\title{
A Feature-Based Model for Part Representation in a DFM Environment
}

\author{
C. Pantoja-Lima*; J. J. Pamies-Teixeira** \\ Universidade Nova de Lisboa - Dep. de Engenharia Mecânica \\ Quinta da Torre, 2825 - Monte da Caparica - Portugal \\ $\mathrm{Tel}+351-1-350$ 0216/0200 Fax + 351-1-2957786 \\ E-mail: $\{c p l, j p t\} @$ uninova.pt
}

\begin{abstract}
This paper describes development and implementation of a feature-based modelling system for the Design for Manufacturing concept. The implementation of the proposed model proves the feasibility of the concepts concerning Design Tools for an integrated product development as necessary in a Concurrent Engineering environment. The characteristics of this system widen the scope of what a Design System can be in the future as the designer can have a control on the design solution considering the implications in the manufacturing phase. Also, it can comply the design solution to the existent technologies because the model provides information on the manufacturing processes involved together with the capability of the machine. The implemented solution is composed by two modules, the Graphical and the Abstract Modules. This paper is focused mainly in the Graphical module, but the whole system is presented and discussed.
\end{abstract}

\section{Keywords}

Design for Manufacture, Design by features, Knowledge-Based Design System

\section{INTRODUCTION}

It has been recognised in the last few years that the design activity is one key point concerning the product development. The conceptual phase of the design determines $75 \%$ of the product cost in the development cycle of a product (Ullman, 1992). This fact strongly stresses the importance of the design phase.

Considering the available support for the designer, it relies upon modelling tools that basically supply geometry modelling, normally at a very low level of abstraction (Chang, 1990) and unlinked to the following activities namely process planning and manufacturing. Nowadays, the use of the so-called concurrent engineering is becoming a need aiming at having a complete and overall view of the implications of the design phase of the product (PamiesTeixeira, 1993). Therefore, the need of a complete information model integrating all the activities of the product development cycle is more and more justifiable specially as a contribution for the implementation of balanced manufacturing systems.

\footnotetext{
*PhD student of Brazilian CNPq research foundation, Electrical Engineering Dep., FCT/UNL

**Associate Professor, Mechanical Engineering Dep., FCT/UNL
} 
Designing a product in a design for manufacture perspective means the need, at least in some phases, to consider the choice and evaluation of manufacturing processes and product manufacturability in order to reach the adequate decisions in terms of the final design solution. One approach leading to a potential solution to this matter is the design by features, in which a workpiece is composed by several features concerning geometry, topology, material, function, processes and validating semantics (Shah and Wilson, 1988; Pratt, 1988; Shah, 1991). Such a system requires the development of an information model providing a good basis for taking decisions about the product while it is still in the form of a model.

\section{CONCEPTUAL MODEL}

The conceptual model of the information is partially depicted in Figure 1. Basically the definition of a workpiece encompasses two groups of information. The left side of the tree, single components, defines the canonical part of the information where all the feature components of the workpiece are defined. The right side, labelled parts, is used to define the topology of the different components within the workpiece considering the process by which they are connected. The single component is defined by its geometry, material type, type of machine where can be processed and a definition of some details of the manufacturing process.

On the other hand, the parts can be defined as simple or complex. Such classification is based on the linking process existing between features belonging to that part. Simple part means the absence of link process in the part; complex means any kind of connecting process involved in the part. The nature of the linking process give us two types of complex parts: a permanent link (welding) defines a composite part; a temporary link (assembly) defines an aggregated part.

This type of classification at this level was done to provide a separation of the semantics of the descendent features. Thus, all the descendent of the aggregated components have an assembly process involved. Further analysis on the part will trigger on the abstract system the rule set relating to the assembly and there will be no need for the system to look for all the universe of rules. With the other branches the principle is the same. As the complete tree of the implemented model cannot be displayed, only a small part of Figure 1 was used just to clarify the above mentioned concepts.

In this model the concept of feature is extended to mean that any component of the information about a workpiece, either with physical representation or not (virtual feature), is treated as a feature. The features defining the geometry are examples of real features. The union is one example of a virtual feature, which doesn't have a physical representation. This feature defines the topology and attachment of features.

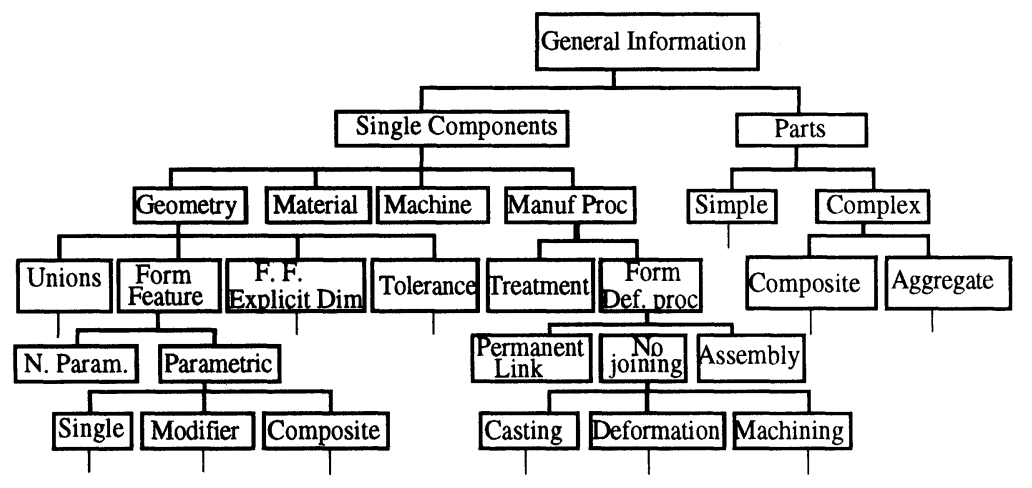

Figure 1 Partial representation of the information model. 
The unions are special features which allow to define the method of connecting two given features and contain the connection process. There will be no associated process if the medium is continuum. The explicit dimension of the geometry is aimed at defining the typical dimensioning in technical drafting. Finally the tolerance feature defines the functional or geometrical tolerance of the feature, associated with the capability of the technological process in force in the organisation. This is done by using instances of the machine feature and manufacturing process feature. A further description of this model is done elsewhere (PamiesTeixeira and Marin, 1992; Pamies-Teixeira, 1993; Cunha and Pamies-Teixeira, 1993; PantojaLima, 1994).

There are several aspects considered by this model. One is to use the model as a tool to exercise the Design for Manufacture that requires (and desires) evaluation of the design in early phases according to existent technologies. Other, is to allow reasoning on the manufacturability and also to use the principles of group technology which with this model can be context dependent. Also, emerging from this model, there is also the possibility of a number of validations on the design, depending upon the application modules. Each application module contains the rules and facts that are essential to the required validation. Design evaluation contains the rules for DFM/DFA analysis while Group Technology is basically a classification and coding system for GT purposes.

\section{SYSTEM DESCRIPTION}

The implemented system is composed by two sub-systems: the graphical sub-system, and the abstract sub-system (also called $K B$ system). The former is responsible by all the interactive work, geometrical visualisation and all kinds of input and output to and from the system. The latter, being knowledge based supported, takes care of all the reasoning according to the application module. The differences in nature of both sub-systems imposed some differences in the feature representation, however the integrity of data according to the explained concepts is fully maintained. The knowledge provided to the KB system is based on operators experience, i.e., all the information they have been used during years is not lost.

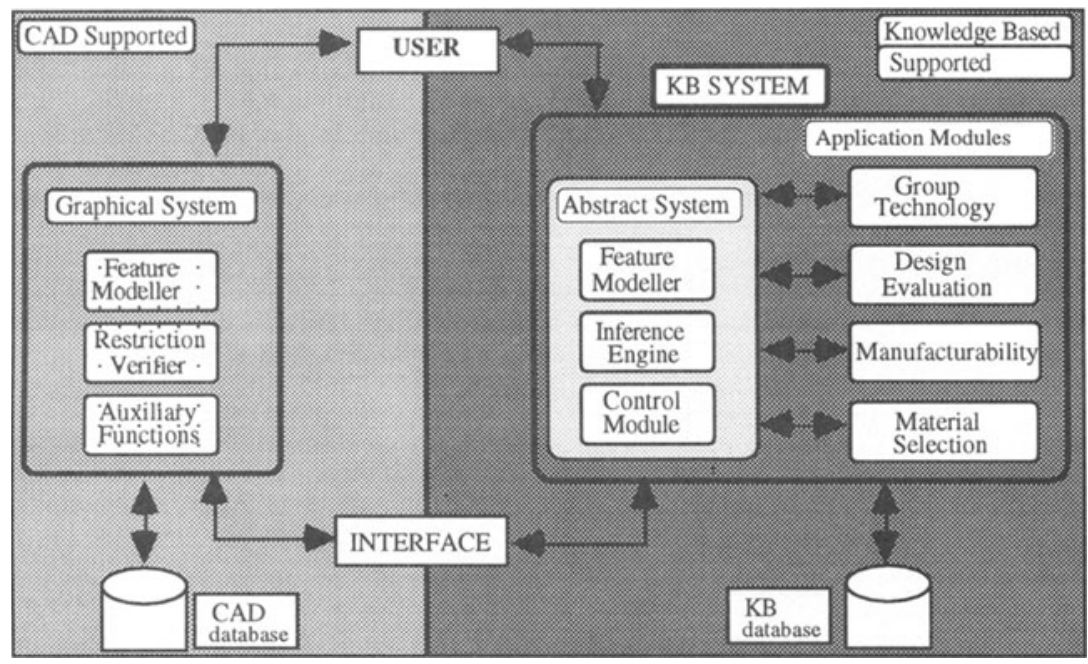

Figure 2 General architecture of the system. 
The general architecture is represented in Figure 2. The abstract sub-system has three main modules which are the kernel of the system. The feature modeller is responsible for the representation of all the knowledge concerning the workpiece. The control module is responsible for interfacing both the user and other sub-systems. The inference engine contains all the basic rules and reasoning of the system. The context dependent rules are contained within the application modules. At the moment, only the group technology, manufacturability and design evaluator are implemented.

\subsection{Abstract Sub-System}

This sub-system being a KB system was implemented with tree main databases: Frame Database, that holds the information tree partially displayed in Figure 1; a Fact Rule Database where both common and axiomatic knowledge are held; Rule Databases where the rules to be applied to solve the queries are implemented. For the sake of readability and maintenance, different fact and rule bases were uses according to the nature of knowledge. On top of this structure an inference engine and a control module was built (the main user interface) which always seek for a response to the queries to the system. The feature modeller is a rule-set which provides the validation of the feature model. The Design Evaluator copes only with simple cases, as base plates for injection plastic moulds.

The current prototype can respond to several situations: Group Technology (classification and coding), Evaluation of Manufacturability (seeking for the best machine and resources within the available machines); Material Selection and/or validation, according to the surface condition or other attributes assigned by the designer.

\subsection{Graphic Sub-System}

This sub-system is functionally divided into three modules: a Feature Modeller, a Restriction Verifier and Auxiliary Functions (see Figure 2) The first one contains and takes care of all representation through the primitive features existent within the modeller. The second one, is responsible for consistency checking about geometry, topology, material, processes etc. Finally the module of Auxiliary Functions provides all the functionality to the user. A set of facilities, in the system operation mode, were designed to give to the user, an easy and comfortable control on it. Hence, it may considered that the full development and the functionality considerations had completely took the human centred aspects into account.

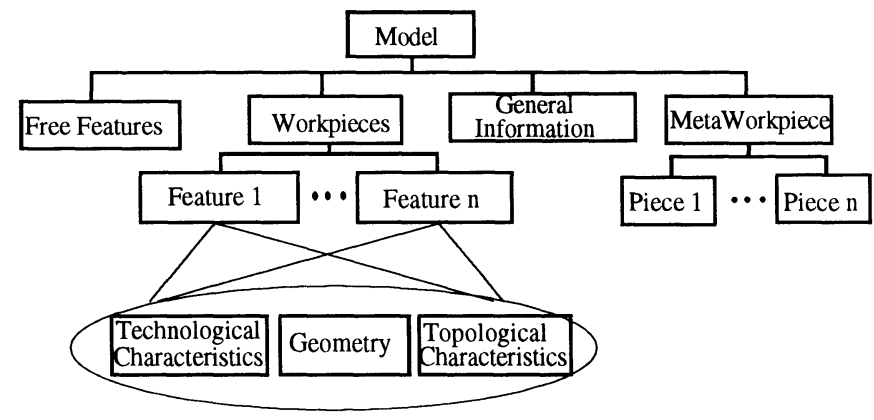

Figure 3 Graphical Entities

The information in the graphic module is represented in the so-called model, which contains all information about a product ( Figure 3 ). There are other entities in the model composition. The Workpiece is the fundamental ground for the model construction, and it is composed by lists of features which encompasses technological, geometrical and topological elements, in 
agreement with the underlying concepts explained earlier. The Free Features have been created to allow the user to place individual features, which do not belong to any workpiece. In some instances this is useful because it allows 'auxiliary constructions'. The General Information entity is just a class to provide the support to the all information which is normally placed in traditional CAD systems as attributes for administrative purposes. The Meta-Workpiece is a temporary entity created only for geometric manipulation purposes. This is very useful for the user and enhances the user friendliness.

A workpiece is structured using two features lists : 1) the union (virtual) feature list, which contains information about topological elements, that is, the knowledge concerning on how the features are linked in order to achieve the final workpiece form (the so-called adjacency relation) 2) the feature list which contains instances of feature objects. As stated early, in this research the concept of feature is not synonymous of form feature. One feature has technological attributes, geometric parameters, form definition and dependency and positioning relationship with other features. The Figure 4 show the implementation view of the feature concept.

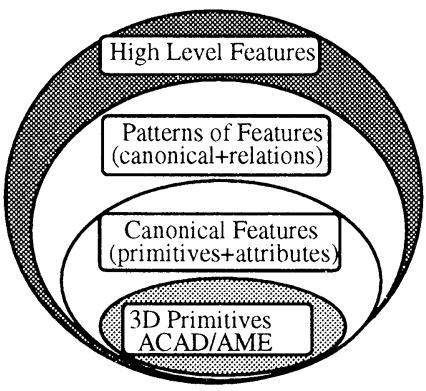

Figure 4 Implementation of features in the Graphic System.

The inner level is represented by the ACAD+AME primitives. They are volumetric entities such as parallelepipeds, cones, cylinders and wedges. This set of primitives added with attributes defining their nature (positive, negative and modifier) and technological characteristics, are the so-called Canonical Set. All of positive, negative and modifier features are physically represented by a solid, although the negatives and the modifiers must have an existential dependency relation on some positive feature. This means that they cannot stand by themselves unless they are fully contained within one or more positive features (a hole is a negative cylinder that must be placed inside some positive feature; fillets and chamfers are examples of modifiers). Patterns of Features are created by adding mathematical relations or functions to the canonical set (such as circular patterns of holes). Finally, the high level features are defined by a set of inter feature relationships, where it is possible, for instance, to derive some parameters and to validate some functional restrictions between them (through-hole, stepped-hole, keyway).

There are three types of relation among features: position, dependency and adjacency. The position relation contains the rules for the positioning and orientation of a given feature with respect to another one to which it is attached. This relation defines the concept of reference and leaf features. This respectively means the features being used as base to instanciate new features as well as the features not used for this purpose. The dependency relation takes care of the existential dependency of a given feature. As mentioned before, this is particularly important for dealing with negative features and modifiers. There are two concepts embedded in this relation: mother and daughter features. The first one is used to specify one feature which contains any part of a negative feature. The second one is used only to negative features which are placed inside one or more positive features. The adjacency relation contains the rules to 
attach two or more features as well as the attachment method and the identification of the faces involved in the connection ( the union feature).

There are several restrictions imposed to the system concerning with geometric, topologic and functional validations. The feature modeller always checks the user actions in order to validate all these aspects of the model. There are four intrinsic restrictions: i) Interpenetrability by which features of the same nature should never intersect to each other; ii) Inaccessibility to avoid closing entry faces of negative features; iii) Existential of first kind to force negative features to be completely embedded in positive features, and iv) Existential of second kind to avoid the very first feature in the model to be negative. The interpenetrability was defined in order to facilitate the treatment executed by the abstract module. The inaccessibility was defined by functional reasons - when a negative feature is attached, one of its face is used as entry face (related by the machining operations), which cannot be closed by the attachment of a positive feature. The two existential restrictions were defined to guide attaching operation of negative features, which must be contained into positive features.

\subsection{Functional Considerations of the Graphic Module}

Each Workpiece has a private coordinate system WCS (Workpiece Coordinate System), and all the geometrical operations performed on the workpiece are referred to that coordinate system. When two features are connected the respective link is established by means of a face and a point from each of the features. The unit vectors through these points must coincide though in opposite directions. The faces involved in this attachment can be totally or partially absorbed by the connection and are classified as virtual or partial. This information is contained in the union feature (seen before).

The features are organised in two libraries. The canonical library containing all the canonical set and the patterns of features (Figure 4). The so-called open-library permits the definition of more general features (macro-features). They are defined using the canonical ones and the relationships rules and restrictions seen before. This concept is one of the more important defined and tested in this research, because it provides a good solution for the classic problem related with the specific set of pre-defined features. With this approach, the set of features available becomes large enough to solve basics requests from the user, and more - this library is customised by the user according to his/her needs, i.e., it is particularly and dynamically customised. Therefore, the power of this concept relies upon the canonical set of features and in the relationship rules and restrictions defined. There is no limit for the number of open libraries which can be useful for the user to create context dependent libraries.

The visual representation chosen was wireframe. It is not the best way considering visual clarity, but is the only one in which was possible - in the platform imposed, to identify the features individually. The solid modeller adopted could not maintain the same identifier of a primitive after a boolean operation, for example. A function to perform all booleans operations existing in the workpiece was offered, so that the final workpiece solid form can be reached. Such function can be executed any time according the user needs.

\section{IMPLEMENTATION}

The Abstract Sub-system is knowledge-based, and it is implemented using an expert shell for DOS operating system. The adopted tool was the Intelligence Compiler, which organises the knowledge by means facts, rules and frames. Frame structure was particularly useful to maintain the paradigm of object oriented programming which was used also in the Graphic Sub-system. This one was developed in the AutoCad R12, using the solid modeller AME and the development environment $A P I$. The software development was performed using $\mathrm{C}++(\mathrm{High}$ $\mathrm{C}$ compiler + PharLap linker) as it was the only one (among the tested ones) capable of dealing with API environment from ACAD+AME. 
The application needed to run in two machines communicating by means of a LAN, since it was not possible to run both ACAD and Intelligence Compiler in the same platform. A software called NetEye was used to allow the user to have in the same screen, the graphical environment and a virtual machine running the $\mathrm{KB}$ system, improving usability and user friendliness. To the user everything happens as if he/she were only in one machine. Although this way of working is not very practical, it was the only way to complete the proposed research in order to comply with the impositions from the sponsor of this research: low cost hardware and commercial software platforms. A schematic representation is given in Figure 5.

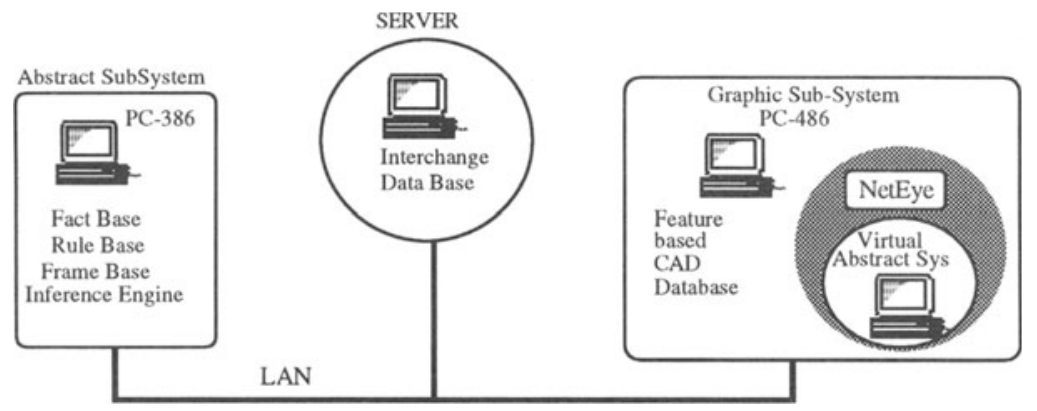

Figure 5 Schematics of the implementation.

The KB system always checks the incoming messages from the Graphic system. These messages are the queries that $\mathrm{KB}$ should answer or give advice. The graphic system uses the interchange database to transfer to KB system, all information about the user request. The virtual machine displays all the messages directly from the KB System.

\section{$5 \quad$ RESULTS AND DISCUSSION}

The implementation of the proposed model proves the feasibility of the concepts related to Design Tools for an integrated product development, as necessary in a Concurrent Engineering environment. The characteristics of this system widen a great deal the scope of what a Design System can be in the future, as on how the designer can have control on the design solution considering the implications in the manufacturing phase. Also, it can comply the design solution to the existing technologies, since the model provides information on the manufacturing processes involved, together with the machine capability. Moreover, due to format and properties of the data created, it makes easier the use of standards like STEP to export information not only concerning geometrical features but also manufacturing features.

However, some difficulties must be shown up in order to point the limitations that an inadequate platform can bring to the normal development. First of all, the main problem met concerns to the development tools compatible with Autocad, namely compilers and linkers. It was not an easy task to overcome the compilation problems and compatibility, since some commercial specifications do not agree with the real packages capabilities. Aside of that, in the $A C A D+A M E$ environment there is no unique and absolute identifier for the solid primitives. This meant that in different sessions the solid identifiers were different. Also there was no possibility of individually identification the primitives involved in boolean operations. This limitations imposed the use of a unusual data structure with implications in the system performance. Moreover, the implementation of fillets and chamfers needed a change of the initial concept because it was not possible to implement them as modifiers but as solid features built by extruding a plane shape through the edge to be modified. 
Finally, taking the current performance of the overall system into account, one can say that it is a "good" prototype. The implementation showed that a much more powerful computer environment was necessary, with an operating system having multitasking facilities. The reasoning process for more complex situations could take several minutes to be executed, which provides a low level of user friendliness. Also, the use of ACAD was very limitative since it obligated us to take a special care to the data structure, so that the model could be retrieved later on. A prototype was installed and tested in one small Portuguese mould maker company with "good" results.

\section{ACKNOWLEDGEMENTS}

We would like to thanks to CNPq for the scholarship, UNINOVA and the Mechanical Engineering Department (FCT/UNL) for the general infrastructure.

\section{REFERENCES}

Ullman, David G. (1992) The Mechanical Design Process, McGraw-Hill.

Chang, T.C.(1990) Expert Process Planning for Manufacturing, Addison-Wesley.

Pamies-Teixeira, J.J. (1993) The Mechanical Design Process, BRITE/EURAM Project 3302, Internal Report, 26 January.

Shah, J.J. and Wilson, P.R. (1988) Analysis of Knowledge Representation and Interaction Requirements for Computer Aided Engineering, Proceedings of ASME Computers in Engineering Conference, San Francisco, California, USA, pp.17-24.

Prattt, M.J. (1988) Synthesis of an Optimal Approach to Form Feature Modelling, Proceedings of ASME Computers in Engineering Conference, San Francisco, California, USA, pp. 263-274

Shah, J.J. (1991) Assessment of Feature Technology, Computer-Aided Design, 23 (5), pp. 331-343.

Pamies-Teixeira, J.J. and Marin, R. (1992) A Feature-Based Design System Development Automation, Proceedings of 2 nd International Conference on Automation Technology, Taipei, Taiwan.

Pamies-Teixeira, J. J. (1993) The Design for Manufacture Applied to the Computer Aided Design of Mechanical Parts, 12th Brazilian Congress for Mechanical Engineering, Brazil.

Cunha, G.D. and Pamies-Teixeira, J.J. (1993) A Feature-Based Model for Design for Manufacturing Knowledge Representation, 12th Brazilian Congress for Mechanical Engineering, Brazil.

Pantoja-Lima, C. P. (1994) Modelling Feature-Based in the Concept of DFM and DFA.. Master thesis, Federal University of Santa Catarina, Brazil.

\section{BIOGRAPHY}

Celson P. Lima received his degree on Computer Science in 1986 and his Master degree in Mechanical Engineering in 1994 at Federal University of Santa Catarina - Brazil. Actually he is taking his Ph.D. at New University of Lisbon. His main interest are: Concurrent Product Design, Design by Features, Design for Manufacture and Concurrent Engineering.

Prof. Dr. J. J. Pamies Teixeira received his Ph.D. in Mechanical Engineering at FCT/UNL, 1985; Master's degree in Mechanical Engineering at MIT in 1976. During several years he have worked for metalworking industries in Design, Manufacturing and Quality while maintaining his position as professor at Mechanical Dep. of FCT/UNL. Also he has developed several researches projects in Robotics, Automation and Design for Manufacturing. 